\title{
Treatment of stove-in chest with 'paradoxical respiration' in peripheral hospitals
}

\author{
FRANCIS SANDOR
}

From Hartlepools Hospital, Hartlepool

The Chyrurgion shal not refuse to cure any hurt of the members contained in the inner part, except ye heart. . . . - Giovanni da Vigo. Practica Copiosa in Arte Chirurgica, Rome, 1514. (Translated by Thomas Gale, London, 1586.)

Stove-in chest presents a particularly serious problem in peripheral hospitals where the facilities of accident centres are not or are only partly available.

In this condition the cardiorespiratory physiology is altered because of the instability of the chest wall. The patients are shocked by pain and loss of blood, but mainly by anoxia and retention of carbon dioxide. They arrive at hospital distressed, cyanosed, and dyspnoeic. The most conspicuous feature is the 'paradoxical respiration', a see-saw movement of the chest, which is aggravated by the respiratory efforts of the patient or by any attempt at expectoration. Associated intra- or extrathoracic injuries may not be obvious.

As immediate action has to be taken, primary resuscitation should not interfere with assessment of the patient or with any treatment that may become necessary. Induction of general anaesthesia appeared to be too hazardous in these shocked and anoxaemic patients. Under local anaesthesia minor procedures were performed in quick sequence to improve their oxygenation and to allow time for their full assessment.

\section{PARADOXICAL RESPIRATION}

The distinguishing feature of stove-in chest is the paradoxical movement of the chest wall, which aggravates all the other effects of the crush injury of the chest.

While the catching pain allows only shallow and rapid respiration, the dead space is increased. Mediastinal flutter may interfere with venous return and elicit distressing reflexes. With increasing distress paradoxical movements become more violent and a vicious circle develops.

At this stage it is irrelevant whether paradoxical respiration is present from the beginning or develops after the patient recovers from shock. The course of events is the same, and it leads relentlessly to complete respiratory failure unless the vicious circle can be broken (case 8; $\mathrm{W}$ indsor and Dwyer, 1961).

Paradoxical respiration demands priority in treat- $\bar{c}$ ment. Various methods have been described for mechanical stabilization of the chest wall, by plating of the sternum, by traction on the ribs, sternum, or soft tissues of the chest wall, and by wiring or intramedullary nailing of the ribs (Jaslow, 1946; Heroy and Eggleston, 1951; Hudson, McElvenny, and Head, 1954; Proctor and London, 1955; Cohen, 1955; Crutcher and Nolen, 1956; Henry, 1957; Robin, 1960; Virshup, 1960; Sillar, 1961).

In these successfully treated cases paradoxical respiration was only reduced; it often persisted for two to three weeks even when considerable weight was used for traction (Robin, 1960). In comminuted fractures of the ribs these methods could not be applied. The number of unsuccessful cases must have been greater and many were probably not reported (Griffiths, 1960).

Survival generally depended on correction of the hypoxaemia, by decompression of a tension pneumothorax and aspiration of the bronchi. Shallow paradoxical respiration was compatible with recovery if the patient was kept well oxygenated.

MATERIAL

During a period of five years, nine patients who had paradoxical respiration came under treatment for $\omega$ stove-in chest. One had a large 'sucking' wound of $\overrightarrow{2}$ the chest, the other eight had closed injuries. Of this latter group two had the 'anterior' and six the 'lateral' type of stove-in chest. These nine patients will be discussed.

Patients who were treated for crush injury of the chest without paradoxical respiration were not included, even when their injuries were otherwise comparable with those of the previous group. Three other patients who died on the way to hospital or 
immediately after arrival and could not have any treatment have also been excluded.'

\section{IMMEDIATE TREATMENT}

In the patient with an open pneumothorax there was no alternative to immediate thoracotomy and repair of the laceration of the lung (case 4).

In closed injuries of the chest the direct treatment of hypoxaemia was considered to be essential and has been performed whenever possible. For this purpose paradoxical respiration was provisionally controlled in the quickest and simplest way. Details are as follows:

1. To relieve pain and distress and as a premedication for the following steps the patients were sedated by morphia, gr. 1/6, and/or Largactil, $50 \mathrm{mg}$., intramuscularly or intravenously with added Daptazol, $30 \mathrm{mg}$. Because of the number of fractures of the ribs, often bilateral, intercostal block was used in only one instance, but was found to be exhausting for the patient (case 6).

2. The chest wall was manually supported over a cotton wool pad during the periods of distress. Whenever necessary and possible, strapping was applied (cases 1, 2, 3 and 6; strapping was not necessary in case 9 and it was not possible to apply it in case 8).

An encircling elastic adhesive bandage was placed horizontally round the lower ribs. At the site of maximum instability a second tour was started and carried obliquely over the front of the chest to the opposite shoulder and from there across the back to its point of origin. This had a remarkable stabilizing effect in the 'lateral' type, did not interfere with the expansion of the other side of the chest, and allowed the insertion of an intercostal tube. In the 'anterior' type strapping was of no avail, mainly because paradoxical respiration was confined to the upper half of the chest (cases 5 and 7).

These first two steps were considered to be no more than first-aid measures that should be applied not only in hospital but wherever the patient is found. Under the effect of sedation and of some stabilization of the chest wall the patient's condition improved. This made the further steps easier.

3. A radiograph of the chest was taken in every case, if only in the supine position. This sometimes gave information of the type and number of the fractures and of intrathoracic displacement of the fragments. To avoid blurring, the films were taken in expiration if possible. In the presence of gross

1 All three patients had various and multiple fractures, but died because of intrathoracic injuries. Necropsy showed rupture of the aorta with immediate exsanguination in two patients; the third had a large para-aortic haematoma and rupture of the diaphragm with intrathoracic prolapse of the liver. surgical emphysema it was often difficult to diagnose the presence of a pneumothorax because the air bubbles within and between the muscle fibres could simulate lung markings. When two films were taken in sequence, using different penetration, an intrapleural space could nearly always be demonstrated.

4. Transfusion was started while the films were being developed.

5. Pneumothorax was treated by underwater seal drainage using an intercostal Malecot catheter (cases 2, 3, 6, 8 and 9). This catheter was also convenient for drainage of a haemothorax (cases 8 and 9). In one case, needle aspiration of the haemothorax proved to be more exhausting for the patient (case 6) than catheter drainage.

6. The bronchi were aspirated by bronchoscopy under local anaesthesia (cases 1, 2, 3, 5, 6, 8 and 9) and under general anaesthesia (case 7). Immediate post-operative bronchoscopies were performed under general anaesthesia (cases 4 and 7). If further bronchoscopies became necessary, local anaesthesia was used (cases 2, 3, 4, 6, 7 and 8). Three was the maximum number of bronchoscopies in any one patient, and tracheostomy was not necessary except in one patient (case 5), who was treated in a cottage hospital where no bronchoscopist was available.

In one case of exceptionally tense surgical (mediastinal) emphysema small incisions were made in the supraclavicular fossae. Oxygen was given continuously through a transnasal, intrapharyngeal tube. This reduced the dead space by delivering oxygen to the entrance of the larynx (Bruce, 1959, personal communication). Penicillin or other antibiotic was started.

\section{PROGRESS AND RESULTS}

Following immediate thoracotomy, the patient with the open injury recovered (case 4).

The two patients with the anterior type of closed injury did not respond well to the first-aid measures. The chest radiographs suggested an intrathoracic injury and a thoracic surgeon was consulted. Both patients underwent immediate thoracotomy; one recovered (case 7) and the other died (case 5).

In the six patients with the lateral type of closed injury the full procedure was carried out. To each step they responded satisfactorily, even dramatically. No more than repetition of these minor procedures later became necessary. Some of them were in a critical, even moribund condition, but they have all recovered (cases 1, 2, 3, 6, 8, and 9).

\section{CASE HISTORIES}

CASE 1 H. C., a man, aged 54, Kirkcaldy General Hospital No. 44820, was a known pneumoconiotic and 
chronic bronchitic patient. On 3 March 1956 the truck on which he was travelling overturned and he sustained a crush injury of the chest with bilateral fractures of the ribs: on the right side the sixth and seventh ribs were broken in the posterior axillary line and the second to fourth ribs in the parasternal line; on the left side the third to seventh ribs in the posterior axillary line and the second to fifth ribs in the parasternal line were broken. He was distressed, dyspnoeic, and cyanotic on arrival at hospital. The left side of the chest was bruised and flattened. Gross paradoxical movements were seen only on the left side.

After sedation and strapping a radiograph showed a small apical haemopneumothorax. He was turned on to his left side. It was later noticed that paradoxical respiration had diminished. He needed one bronchoscopy and made an uneventful recovery. He was discharged eight days after the accident.

Comments It was believed that incidental displacement and 'locking' of the ribs occurred at some stage (see also case 9).

CASE 2 W. M., a man aged 74, Kirkcaldy General Hospital No. 57294, had been under medical treatment for productive cough, attacks of dyspnoea, and pneumoconiosis, for which he drew a $50 \%$ disability pension.

On 28 April 1957 the bus on which he was travelling overturned and he sustained fractures of the second to eleventh left ribs in the scapular line and of the second to seventh left costal cartilages. On arrival he was nearly moribund. There were extensive bruises and gross surgical emphysema over the whole of the left side of the chest, which showed violent paradoxical movements. He was fibrillating and the pulse was hardly palpable. Blood pressure was $80 / 60 \mathrm{~mm}$. Hg. After sedation and strapping the left tension pneumothorax was drained using a catheter connected to an underwater seal; air escaped under pressure.

There was a dramatic improvement in his condition. His distress was relieved and paradoxical movements were diminished. The pulse became stronger, though he was still fibrillating. With assistance he was able to expectorate copious amounts of mucopus. On 29 April he became suddenly distressed and cyanosed, with gross deterioration of the paradoxical respiration. He became 'bubbly' and collapsed, losing consciousness. The radial pulse was not palpable and $2 \mathrm{ml}$. of coramine was given intravenously. The pulse returned and bronchoscopy was immediately performed. The trachea and stem bronchi were almost full of viscid mucopus which darkened the light of the bronchoscope. After the bronchi had been cleared he regained consciousness, paradoxical movements of the chest wall became shallow and the pulse stronger. Because of general weakness, he was unable to clear his bronchi adequately and needed a bronchoscopy after two days and also after another five days. Gradually he became stronger. Paradoxical respiration entirely disappeared after a further five days. On 13 May 1957 he was febrile and a small fluid level was seen on the right side when a radiograph of the chest was repeated. He was transferred to the Regional Thoracic Unit in Edinburgh as a lung abscess was suspected. With physio- $\square$ therapy alone he recovered and was discharged home.

Comments After this case, bronchoscopy was routinely included in the initial treatment.

CASE 3 F. T., a man aged 29, Kirkcaldy General Hospital No. 59721, was involved in a car accident on 25 August 1957, and sustained multiple fractures of the $\vec{\omega}$ right scapula, the right clavicle, and of the following ribs. On the right side the first to tenth ribs were broken in the posterior axillary line and the second to fourth ribs in $\stackrel{x}{-}$ the parasternal line; on the left side, the second to fourth $\vec{\infty}$ ribs were broken in the posterior axillary line. He arrived iv in great distress, having gross surgical emphysema over $\vec{\overrightarrow{ }}$ the right side of the chest and violent paradoxical move- $\sigma$ ments on this sidz. After sedation and strapping the radiograph of the chest showed a tension pneumothorax which was drained by intercostal catheter and under $\overleftarrow{c}$ water seal. At bronchoscopy a great deal of blood and $\overline{\overparen{D}}$ mucopus was aspirated. Visibility was not good, but as $\vec{D}$ far as possible the segmental orifces were also aspirated. He improved greatly. Paradoxical movements were still present but were much diminished. On 27 August he had $\square$ an incident of pulmonary oedema which necessitated a hurried bronchoscopy. There was still much blood in the bronchi. Improvement was not as marked after this bronchoscopy as in the first instance. Paradoxical $\frac{2}{\Phi}$ respiration remained of considerable intensity. A radio- $\varrho$ graph of the chest now showed collapse of the middle and $\overrightarrow{\overline{0}}$ lower lobes of the right lung.

On 31 August bronchoscopy was repeated, and much mucopus was aspirated, but there was no more blood in $\frac{\overrightarrow{0}}{0}$ the bronchi. Thereafter recovery was slow but uninterrupted. Paradoxical movements disappeared entirely after two weeks. The lung expanded, but there were still opacities in the lower lobe when he was discharged four $\stackrel{\times}{\circ}$ weeks after the accident.

Comments Considering the great amount of blood that was aspirated at the first bronchoscopy, this $\frac{\text { 을 }}{3}$ treatment should have been repeated on the following day. Pulmonary collapse might then have been avoided.

CASE 4 S. M., a man aged 49, Kirkcaldy General Hospital No. 38191, was hit by a falling lever whilst $\mathrm{O}$ working in the pit on 2 November 1957. He sustained a N large penetrating wound on the right side of the chest ${ }_{\sigma}^{\omega}$ with severe comminution of the fourth to eighth ribs, mainly laterally. On arrival he was in a critical condition,, greyish, cyanosed, and dyspnoeic, with gross paradoxical respiration on the right side. There was a $40 \mathrm{~cm}$. long? wound along the sixth intercostal space, which had $\square$ opened the pleura in its entire length. The wound was $\underset{P}{ }$ soiled with dirt and pieces of coal; it had been covered $\overparen{\Phi}$ with Vaseline gauze, which had acted as an expiratory $\frac{\vec{D}}{\mathrm{D}}$ valve; the dressing sealed off the wound on inspiration, $\frac{a}{\sigma}$ while air was hissing out during expiration. But for this skilful first-aid dressing he would not have survived the 
journey to hospital. He was taken forthwith to the theatre.

At induction of anaesthesia the patient was immediately turned on to his left side, and a deep laceration of the lung was packed to control the air leak. Thereafter the anaesthetist had no difficulties with ventilation. The patient's colour immediately improved. The wound of the chest wall was excised. The pleura was soiled in the same degree as the chest wall, especially on the diaphragmatic surface; it was cleared and a tear of the diaphragm repaired. The pack was then removed. The lower lobe was seen to be almost completely divided from its base to its apex and the two parts were separated. A tear at the side of the lateral basal bronchus was repaired. Two smaller bronchi were completely transected, and were closed by suture. The wound of the lung was sutured. Except for the lateral basal segment, which remained partially collapsed, the lung could then be inflated. A pleural drain was inserted through a stab wound. Closure of the chest wound was difficult because of the comminution of the ribs and tears of the soft tissues. The loose and flail parts of the ribs were brought into alignment. By including these fragments into the sutures which united the soft tissues, it was possible to keep them aligned, and the wound was closed. Spontaneous respiration was resumed, and considerable paradoxical movement was seen. Paradox was substantially controlled by strapping, as described above. At bronchoscopy blood was aspirated from both bronchi. When the patient recovered from anaesthesia, his colour was good and respiration was quiet.

On 3 November he was cyanosed and paradoxical respiration had increased. A radiograph of the chest showed a large apical space and atelectasis of the right lung. There were opacities in the left lung. A Malecot catheter was inserted into the pleural space through the second intercostal space without disturbing the strapping.

A great deal of blood was aspirated through the bronchoscope, mainly from the right bronchi. He was greatly relieved, and respiration became quiet.

On 4 November he needed another bronchoscopy to control bronchial retention. He was relieved and recovery was then uninterrupted. Paradoxical movement disappeared after five days. He was transferred to a convalescent home two weeks after the accident and went home two weeks later.

Comments Because of the 'softening' of the chest wall, mechanical stabilization was not possible at thoracotomy. His injury was converted to a closed stove-in chest, which was treated with strapping, drainage of the pneumothorax, and bronchoscopy.

CASE 5 H. L., a man aged 63, was admitted to Adamson Memorial Cottage Hospital, Cupar. Cn 6 December 1957 he was pinned between a lorry and the parapet of a bridge and sustained severely comminuted fractures of the first to sixth right ribs laterally and at their cartilages. The first to third ribs were also broken posteriorly. Gross surgical emphysema extended over the right side of the chest up to the face, and violent paradoxical move- ments were seen, mainly in the upper half of the chest wall. He was very shocked, cyanosed, and dyspnoeic. After sedation and manual support of the chest wall, a radiograph of the chest showed a shallow pneumothorax, and that the ends of the broken ribs were protruding deeply into the thorax. The violent paradoxical movements were difficult to control even by manual support. He began to be 'bubbly'. A laceration of the lung was suspected and a thoracic surgeon was immediately consulted for the further management of the case. 'The patient was taken to theatre and a bronchoscopy was performed under local anaesthesia. The patient's condition rapidly deteriorated. General anaesthesia was started, but it was not possible to inflate the lungs because of the air leak. Increase of the surgical emphysema was noted on positive pressure respiration. The pulse was absent. The patient was turned on to his left side, and thoracotomy was performed. A deep laceration of the right lung was packed. The lungs could be inflated and the colour and circulation immediately improved. No injury of the bronchus was found. The laceration of the lung was loosely sutured. Two intercostal tubes were inserted through separate stab wounds. The severely comminuted ribs were brought into alignment and kept there by including them in the sutures, which united the soft tissues. Tracheostomy was performed, and the bronchi were cleared of blood and mucopus. When spontaneous respiration was resumed, serious paradoxical movements were still present. Strapping was applied with little effect. The patient recovered from anaesthesia and respiration was not laboured. The bronchi were regularly aspirated.'

On 7 December the patient's colour was satisfactory. Respiration was paradoxical but quiet. Radiography of the chest showed expansion of the lung. The bronchi were kept dry. In the afternoon he collapsed and died within hours.

Necropsy showed advanced atheroma and stenosis in all the branches of the coronary arteries. The anterior descending branch was occluded by recent thrombus. There was haemorrhagic infiltration of the right lung which was practically airless. There were gastric contents in the trachea and in both bronchi, in the stem bronchus only, on the right, but on the left side also in the distal bronchi of both lobes.

Comments Aspiration of gastric contents might have been only a terminal event (Gardner, 1958) but the possibility that aspiration initiated the fatal collapse could not be excluded. R egular aspirations through the cannula did not prevent atelectasis of the right lung.

CASE 6 On 2 May 1959 J. M., a man aged 44, Hartlepools Hospital No. 88345 , fell $30 \mathrm{ft}$. $(9.14 \mathrm{~m}$.) and sustained concussion, a deep laceration of the scalp, comminuted fractures of both condyles of the left tibia, of the head of the left fibula, and of the left clavicle, a fracture of the sternum at the level of the third rib, fractures of the third to eleventh left ribs in the posterior axillary line and of the fifth to ninth left ribs in the nipple line. 
He was deeply shocked, confused, cyanotic, dyspnoeic, and in a state of cerebral irritation. There was surgical emphysema over the whole of the left side of the chest which moved paradoxically. The pupils were fixed and dilated. The pulse was hardly palpable. Blood pressure was $70 / 40 \mathrm{~mm}$. Hg. After sedation and strapping, a plasma transfusion was started and a left tension pneumothorax drained in the usual way. His condition and colour improved, but his blood pressure did not rise from its original level although $2 \mathrm{ml}$. levophed was added to the plasma transfusion. There was a delay in arrival of blood and he became restless. To exclude stimulation by pain, an intercostal procaine block was given in stages; this was done because he became immediately distressed and cyanosed whenever he was slightly turned on to his other side.

After the first pint of blood had been given his blood pressure rose to $100 / 70 \mathrm{~mm}$. $\mathrm{Hg}$. By that time he was 'bubbly'. A great amount of mucopus was aspirated from both bronchi and his blood pressure rose to $130 / 70$ $\mathrm{mm}$. $\mathrm{Hg}$. Cyanosis and distress disappeared, he became fully conscious, and the pupils reacted normally. Four pints of blood were given and the wound was sutured; but the fractured left leg was only positioned between sandbags. Paradoxical movements became shallow.

The next day he remained well and the lung expanded. In the evening he had an incident of pulmonary oedema, which necessitated a hurried bronchoscopy. His progress was rather slow but continuous. The intercostal tube was removed on the sixth day. Paradoxical movements were still marked.

On 11 Nay he became distressed, cyanosed, and dyspnoeic with a marked increase of the paradoxical movements. The trachea was deviated to the left. A radiograph of the chest showed a completely opaque left hemithorax due to a haemothorax and collapse of the lung. He was drowning in his secretions, and a great amount of mucopus was aspirated. During the relief that followed, a full length of plaster of Paris bandage was applied to the left leg. Aspiration of the pleural cavity was postponed until the following day, when two pints of heavily bloodstained fluid were withdrawn. Repeated radiographs still showed residual opacities that were slow to clear, and paradoxical movements were present during the third week, though much reduced. He was transferred to the Regional Thoracic Unit, Shotley Bridge Hospital, on 25 May. He recovered on physiotherapy alone, and was discharged home on 4 June.

Comments In my opinion, the main cause of shock was hypoxaemia. When the oxygen-carrying power of the blood had been improved by blood transfusion, and when oxygenation became possible by clearing his bronchi, the blood pressure returned to normal. The harmful effects of hypoxio-hypercapnia on the central nervous system were concurrent in this case: when this had been corrected, consciousness returned and the pupils reacted normally. Delay in repeating bronchoscopy allowed 'wet lung' with all its consequences.
CASE 7 On 19 June 1959 M. L., a boy aged 16, Hartlepools Hospital No. 89133, was crushed underground between runaway tubs and sustained a fracture of the left superior pubic ramus and the left ulna and severe comminuted fractures of the right upper ribs. The right side of the chest was flattened and the mark of a wheel could be seen running across the front of the chest from the right shoulder obliquely down to the epigastrium. He was distressed, shocked, cyanosed, and dyspnoeic with particularly violent paradoxical movements of the upper two-thirds of the front of the right side of the chest. There was no clinical evidence of a pneumothorax, though there was surgical emphysema over the right side of the chest.

After sedation and manual support of the chest, a i radiograph showed a dense opacity in the right mid-zone, $\vec{\overrightarrow{ }}$ but also that the broken ribs had been driven deeply $\vec{\sigma}$ into the thorax. A thoracic surgeon took over the further management of the case.

'Bronchoscopy under general anaesthesia was followed $\bar{c}$ by thoracotomy through the fifth intercostal space. Two pints of blood were removed from the pleural cavity. A subsegment of the anterior part of the upper lobe was $\vec{\varphi}$ almost entirely torn off and was hanging on a narrow stalk. This was removed and the wound of the lung sutured. The protruding ends of the ribs were elevated and bent in such a way that the lower was bent upwards and fixed to the first stable upper rib by suture, while the upper was bent down and similarly fixed to the first stable lower rib. These two ribs overbridged the gap in a criss-cross fashion. Two tubes were inserted through stab wounds and the wound was closed.'

The patient needed a bronchoscopy on the following day and recovered on physiotherapy thereafter. Paradoxical movements disappeared on the sixth day. After the fracture of the pelvis had healed three months later, he was discharged home. The residual deformity is hardly seen, except on a radiograph of the chest, and does not $\stackrel{\square}{x}$ cause any disability. $\mathrm{He}$ is working as a miner.

Comments In the three patients (cases 4,5 , and 7) the comminuted fractures were caused by direct impact or 'inward thrust' (Barrett, 1960). Strapping is of no avail in the 'anterior' stove-in chest, especially in the upper half of the chest. The bulk and $\frac{7}{0}$ contractions of the pectoralis major and the latissimus dorsi take up all the pressure. Internal displace- $\mathrm{N}$ ment of the broken ribs may necessitate thoracotomy $N$ even when the patient is on positive pressure respira- N tion because the protruding ribs cause recurrent $\omega$ pneumothorax (Windsor and Dwyer, 1961).

CASE 8 R. W., a man aged 47, Hartlepools Hospital No. 97803, was admitted on 27 May 1960, after a car had mounted the pavement where he was standing and hit $\bar{O}$ him. He sustained a fracture dislocation of the twelfth $\overrightarrow{\mathbb{D}}$ on the eleventh dorsal vertebra with immediate complete $\frac{\rho}{\Phi}$ paraplegia. In hospital he was resuscitated, and skin $\varrho$ traction was applied on both legs. During the early hours of the next morning, he became gradually restless, $O$ 
dyspnoeic, and cyanosed. Surgical emphysema developed on the right side of the chest and spread over the left side and also to the neck. Paradoxical movement then started on the right side but became bilateral; and later this became even more marked on the left side, mainly in the lower half of both sides.

When seen, these movements were violent on both sides, and surgical emphysema extended symmetrically from the face down to the level of the symphysis. The patient was extremely distressed. The trachea was deviated to the left. A fracture of the sternum and of the costal cartilages could be palpated on both sides. After manual support of the chest wall and sedation, a Ryle's tube was passed because of acute dilatation of the stomach. A radiograph of the chest was taken in the supine position. A fracture of the sternum at Ludwig's angle and at the level of the fourth intercostal space, and fractures of the fourth to tenth left ribs and of the third and fourth right ribs, were shown. The film was blurred and difficult to read because of the gross surgical emphysema, but the edge of the right lung could be seen, confirming the diagnosis of a right tension pneumothorax. Air escaped with pressure when this pneumothorax was drained with underwater seal. The trachea became central. At bronchoscopy much mucopus was aspirated from the trachea and the right stem bronchus and segmental bronchi. There was a kink at the carina and the bronchoscope could not enter the left bronchus which was blindly aspirated and more mucopus removed. The patient improved, but he remained distressed and the paradoxical movements were still considerable. Because of these features, together with the bronchoscopic findings, a left pneumothorax was suspected. On the table a more penetrating film was taken, which clearly showed a space on the left side. This was immediately drained through the fourth intercostal space with underwater seal. The left bronchi were aspirated by a bronchoscopic catheter and improvement was dramatic. The patient became comfortable, and paradoxical movements were shallow and quiet without any support.

On 29 May surgical emphysema had diminished, respiration remained quiet, and he began to expectorate with assistance. Satisfactory expansion of both lungs was shown on the radiograph of the chest, though there was still a small space on the right side. Some air was bubbling through the right pleural tube. In the afternoon he was turned on to his side for treatment of the pressure areas and he suddenly became very distressed, cyanosed, and dyspnoeic, with violent paradoxical movements of the chest wall. Surgical emphysema rapidly spread and within minutes it extended from the scalp down to the knees, where only the bandage prevented its further spread. He could not open his eyes and the scrotum was ballooned. Surgical emphysema was particularly tense over the neck and sternum, where the soft tissues were elevated at least 3 in. $(7.62 \mathrm{~cm}$.) from the bone. There was a differential cyanosis of the face and neck, which were almost black, suggesting a tense mediastinal air leak. An overlooked fracture of the trachea or either bronchus, a recurrent pneumothorax on any or both sides were possibilities. The patient's condition was critical.
After intravenous sedation with added daptazol, small incisions were made in both supraclavicular fossae. Air hissed out of the wounds, and when the spontaneous escape settled, more air was expressed manually from the subcutaneous tissues. Differential cyanosis disappeared. Two radiographs with different penetration were taken in sequence and compared. Recurrence of the left pneumothorax was confirmed on the more penetrating film. This pneumothorax was immediately drained in the second intercostal space. When bronchoscopy was performed, no kink at the carina was seen; both bronchi could be aspirated under vision. The patient, who had been moribund shortly before, became comfortable. Respiration was quiet and no further support of the chest wall was needed. Traction on the legs was discontinued and turning of the patient suspended for 48 hours.

On 30 May a right haemothorax ( 26 oz.) was drained with underwater seal. The patient now had two intercostal tubes on each side. His recovery was uninterrupted and paradoxical movements entirely disappeared after the twelfth day. A radiograph of the chest was near normal when he was transferred to the Spinal Injuries Unit, Lodge Moor Hospital, three weeks after the accident. The paraplegia did not improve and he is now leading an armchair life.

Comments Delayed onset of paradoxical respiration has also been recorded by Virshup (1960) and by Windsor and Dwyer (1961). The tense mediastinal emphysema compressed the veins of the neck and head, a rare complication, which necessitated treatment by incisions in the neck. The trachea was deviated to the left, when the patient had a bilateral pneumothorax, and was not palpable at all when the left pneumothorax recurred. On both occasions only the penetrating film of the chest made the diagnosis possible. Without decompression of the pneumothoraces, relief would not have been possible, and, in my opinion, induction of general anaesthesia might have been fatal.

CASE 9 J. T., a man aged 43, Hartlepools Hospital No. $2627 \mathrm{Z}$, was in the pit on 1 January 1961 when he was struck by a heavy fall of coal and stone. He sustained fractures of the right clavicle, of the sternum, of a right cervical rib, of the first to eighth right ribs in the posterior axillary line, and of the cartilages of the second to fifth right ribs. Depression of the right anterior chest wall could be seen and palpated. Paradoxical movements were evident on the right side, where there was surgical emphysema which extended to the face. The trachea was deviated to the left. After sedation and manual support of the chest wall, the right tension pneumothorax was drained in the usual way. Copious amounts of mucopus were aspirated from the trachea and both bronchi, although there was no 'clinical evidence' of bronchial retention. Relief was immediate. There were hardly any paradoxical movements. The ribs had displaced some time during treatment and 'locked' in that position. Two days later a right haemo- 
thorax was drained under water seal. Recovery was uneventful and he was discharged home two weeks after the accident. He is working as a miner. The chest expansion is at present $3 \frac{1}{2}$ in. $(8 \cdot 8 \mathrm{~cm}$.).

\section{DISCUSSION}

Crush injury of the chest may be fatal without injury of the lung, by bronchial retention, pulmonary oedema, haemothorax, or pneumothorax. This is especially true when a segment of the chest wall becomes mobile. Paradoxical respiration accelerates the development of asphyxia; its control is essential in order to treat the other effects of the injury, which may become equally fatal.

The type of the fractures allows some conclusions as to the severity of the trauma. In a particularly severe impact the 'inward thrust' causes local comminution of the ribs. When the impact is less violent, the fractures occur first at the weakest points of the ribs by 'outward burst' before the direct fractures. More serious intrathoracic injuries can be expected by more violent trauma; the type of the fractures is therefore important in the assessment of the patient's injuries.

Data from the literature, and experience in this series, suggest that the more the stability of the chest wall has suffered, the more difficult is the problem of definitive mechanical stabilization, even by thoracotomy. At the initial treatment it is logical to aim at a quick provisional control of the paradoxical respiration for the period, during which hypoxiohypercapnia is corrected by as early and as complete expansion of the lungs as possible. The definitive treatment can be better decided by the response of the patient, especially if he remains conscious. The 'splinting' effect of the expanded lungs (Avery, Mörch, and Benson, 1956), incidental locking of the ribs, and relief of the respiratory distress reduce the violence of the paradoxical movements.

The following points deserve further consideration. The frequency of associated pneumothorax has not been emphasized even in recent literature. In this series there was only one patient (case 7) who had not had a pneumothorax, and another (case 1) whose pneumothorax was localized. In the remaining seven patients pneumothorax was a vitally important complication. It is probable that in many fatal cases the cause of death was undiagnosed and untreated pneumothorax. Radiography of the chest is therefore essential.

For clearing of the bronchi, aspiration through an endotracheal tube (Barrett, 1960; Griffiths, 1960) or through a tracheostoma (Hulman, 1957) was recommended. In this series, general anaesthesia was used only for thoracotomies (cases 4, 5, and 7). Local anaesthesia has definite advantages, especially in the $\stackrel{\overrightarrow{\vec{F}}}{\overrightarrow{7}}$ assessment of associated head injuries or blunto abdominal injuries. The lungs can be cleared better $\underline{0}$ by bronchoscopy when the segmental bronchi can be $\frac{\bar{c}}{\frac{5}{\sigma}}$ aspirated under vision. Tracheostomy has its own $\stackrel{\varnothing}{\varnothing}$ complications, even when performed in a 'list' case (Aird, 1957; Lewin, 1959), and may not prevent atelectasis of the lung even with the greatest care. (Griffiths, 1960; case 5).

However extensive the fractures (cases 2,6 , and $\stackrel{\omega}{\omega}$ 8), management of the paradoxical respiration $\vec{x}$ became possible by simple means when there was no severe comminution. On the other hand, the three ${ }_{i}^{\infty}$ patients who had severely comminuted fractures needed thoracotomy, even though the unstable $\vec{\sigma}$ segment was smaller. The type of the fractures has a definite bearing on the management of the case, more than the clinical condition of the patient. Even $ᄃ$ moribund patients recovered by treatment with $\underset{\varnothing}{\frac{1}{\sigma}}$ minor procedures in the previous group.

It may be stated that all the pathological features of stove-in chest demand immediate treatment. The multiple fractures of the thoracic cage produce a variable degree of instability, but tension pneumothorax and/or bronchial retention also play their part at least as contributory initiating factors in triggering off a vicious circle.

The sooner respiratory distress develops, the $\overrightarrow{\vec{O}}$ sooner will paradoxical respiration become the 3 dominant disability by its increasing intensity, and the clinical picture becomes the same. If hypoxio-? hypercapnia is corrected or prevented from developing at an early stage, the initiating of this vicious circle is confined to the paradoxical respiration as $\widetilde{x}$ determined by the anatomical damage to the $\frac{5}{3}$ thoracic cage.

This damage may be so severe that paradoxicalo movements can be controlled only by intermittent positive pressure respiration, which eliminates the음 negative phase of the respiration. But in other cases, and they were in the majority in this series, this may․ㅡ․ not be required.

The response of the patients to each step of this procedure gave a clear indication as to the group to which they belonged. Some improvement followed inc each case after the first-aid measures. If there was no evidence of major intra- or extrathoracic injury, aso ascertained by immediate radiological examination, $\mathbb{\infty}$ and the patient's response was satisfactory, the full ${ }^{\circ}$ procedure was carried out. For the residual para- -0 doxical respiration, strapping was a sufficient treat ment in our cases, but, in others, traction by one of the described methods may be necessary.

When the patient's response was not satisfactory $\overline{2}$ the procedure outlined above was not pursued. Im 
particular, in open pneumothorax, operation is the primary and also the definitive treatment. In our case there was such a severe comminution of the ribs with tears of the soft tissues present that mechanical stabilization was not possible at thoracotomy. As the damaged area was confined to the lateral side of the lower half of the chest, strapping was efficient in controlling the paradoxical movements. Alternatively, intermittent positive pressure respiration would have been necessary for a prolonged period.

Two patients with closed injuries had a similar damage to the chest wall. Rapid alternation of ballooning and deep retraction of the unstable areas caused great distress to the patients and this was only slightly relieved by the first-aid measures; even manual support of the chest wall involved technical difficulties. Intrathoracic displacement of the fragments was shown on the films of both patients. At this stage, full details could be given to the thoracic surgeon who was immediately contacted. In the three cases induction of general anaesthesia was performed in the presence of the surgeon.

In hospitals where communication with a thoracic unit is more difficult, drainage of even a shallow pneumothorax (case 5) or of the haemothorax (case 7) and bronchoscopy would have been necessary. In our cases the slight improvement was sufficient to keep the patients in reasonable condition until a thoracotomy could be done, a few hours after the arrival of the patients at the hospital. In the other six patients the fractures were extensive, multiple, often bilateral, but not comminuted. The unstable segment moved en bloc and, even when the whole of the hemithorax moved paradoxically (cases 2 and 6 ), this could be reduced by simple measures with significant relief to the patient. Immediate decompression of the tension pneumothorax (cases 2, 3, 6 and 9), which was necessary on both sides in one case (case 8 ), brought about a dramatic relief with great reduction of the paradoxical excursions in all but one patient (case 6), who was still unconscious, but even here the improvement was very marked. Bronchoscopy brought further great improvement in all patients and this was dramatic in case 6 , where bronchoscopy had been delayed because of shock. Consciousness returned after bronchoscopy.

Thorough and early clearing of the bronchi is essential, even when there is no 'clinical evidence' of bronchial retention. When bronchoscopy was delayed (cases 2 and 6), or not thorough because of bad visibility (case 3), 'wet lung' or pulmonary collapse followed, which demonstrated to me the role of bronchial obstruction in initiating a vicious circle.
In my opinion, induction of general anaesthesia is particularly dangerous in the presence of a tension pneumothorax (case 5) (Hamelberg, Mentges, and Dindot, 1960). The air leak may increase on positive pressure respiration, and inflation of the lung may not be possible (Griffiths, 1960). When the bronchi are full of secretions, oxygenation may prove very difficult (Windsor and Dwyer, 1961). In a case mentioned by Waterman (1961), intubation was impossible because of rupture of the trachea, and cardiac arrest occurred because of anoxia. Only immediate and simultaneous bronchoscopy and open cardiac massage saved that patient.

Decompression of a pneumothorax and bronchoscopy should be performed before the patient is anaesthetized. Induction will then be safer, but often anaesthesia will not be necessary.

Tracheostomy is indicated in a deeply unconscious patient, or when too frequent bronchoscopies become necessary, or when there is no bronchoscopist available. In one patient (case 5), the latter was the indication for tracheostomy but this did not prevent atelectasis of the lung. Balancing the pros and cons of tracheostomy and bronchoscopy, it has also to be considered that after the latter the patients remain conscious with an intact glottis. They can co-operate at an early stage in expectoration. After tracheostomy the patients are unable to expectorate in their most critical period and retention of the secretions may occur, unless the bronchi are regularly inspected to assure complete emptying (Logan, 1962, personal communication).

\section{CONCLUSIONS}

Treatment of anoxaemia, i.e., of all the three factors, paradoxical respiration, pneumothorax, and bronchial retention, has to be performed at the primary respiratory resuscitation. While for some patients general anaesthesia and intermittent positive pressure respiration, with or without thoracotomy, remain the only treatment, a number may recover, even from a desperate state, with treatment with relatively minor procedures. Quick performance of these procedures will allow a better selection of those who need additional treatment. Local anaesthesia allows a better assessment of associated injuries and also makes possible the active co-operation of the patient at an early stage.

By including a period of thoracic surgery in the routine surgical training, performance of these minor procedures would be common knowledge. It may be worth while to give a wider trial to this method 'for Nature, by the Chyrugion's side worketh 
so well that oftentimes it bringeth to passe that seemeth impossible'. (Da Vigo, 1514).

I would like to thank Professor A. G. R. Lowdon for his help and encouragement in the preparation of this paper; Mr. A. Logan, for the years in his unit during which I learned the principles applied in this treatment; Mr. P. R. Walbaum and Mr. W. C. Barnsley for performing thoracotomies in cases 5 and 7, respectively, and for their permission to include these cases.

The patients were admitted under the care of $\mathrm{Mr}$. J. W. M. Sutherland and Mr. W. Drummond (Kirkcaldy Geneial Hospital), and Mr. H. M. Grant, Mr. W. Irving, and Mr. A. Webb-Jones (Hartlepools Hospital), to whom I am grateful for the facilities to investigate and treat these patients.

I would like to thank the theatre staff, the nursing staff, and the radiology and physiotherapy departments of both hospitals for their enthusiastic help.

\section{REFERENCES}

Aird, I. (1957). A Companion in Surgical Studies, pp. 442, 443, 594. Livingstone, Edinburgh.

Avery, E. E., Mörch, E. T., and Benson, D. W. (1956). J. thorac Surg., 32, 291.

Barrett, N. R. (1960). Lancet, 1, 293.

Cohen, E. A. (1955). Amer. J. Surg., 90, 517.

Crutcher, R. R., and Nolen, T. M. (1956). J. thorac. Surg., 32, 15.

Da Vigo, G. (1514). Practica Copiosa in Arte Chirurgica, Rome. Translated by Thomas Gale, London, 1586.

Gardner, A. M. N. (1958). Quart. J. Med., 27, 227.

Griffiths, H. W. C. (1960). J. roy. Coll. Surg. Edin., 6, 12.

Hamelberg, W., Mentges, W. F., and Dindot, J. V. (1960). J. Amer. med. Ass., 174, 1400 .

Henry, L. (1957). Brit. med. J., 2, 339.

Heroy, W. W., and Eggleston, F. C. (1951). Ann. Surg., 133, 135.

Hudson, T. R., McElvenny, R. T., and Head, J. R. (1954). J. Amer. med. Ass., 156, 768.

Hulman, S. (1957). Lancet, 1, 454.

Jaslow, I. A. (1946). Amer. J. Surg., 72, 753.

I.ewin, W. (1959). Proc. roy. Soc. Med., 52, 409.

Proctor, H., and London, P. S. (1955). Brit. J. Surg., 42, 622.

Robin, G. C. (1960). Lancet, 1, 26.

Sillar, W. (1961). J. Bone Jt Surg., $43 B, 738$.

Virshup, M. (1960). J. thorac. cardiovasc. Surg., 39, 579.

Waterman, D. H. (1961). Ibid., 42, 297.

Windsor, H. M., and Dwyer, B. (1961). Thorax, 16, 3. 